\title{
Investigating the effect of increased heatsink temperature and packaging-induced strain in a single emitter laser using a laser diode simulation/emulation tool
}

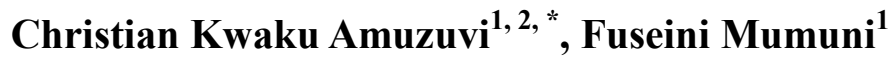 \\ ${ }^{1}$ Department of Electrical and Electronic Engineering, University of Mines and Technology, Tarkwa, Ghana \\ ${ }^{2}$ Photonic and Radio Frequency Engineering Group, Electrical Systems and Optics Research Division, Faculty of Engineering, \\ University of Nottingham, Nottingham, NG7 2RD, United Kingdom
}

\section{Email address:}

ckamuzuvi2000@yahoo.com(C. K. Amuzuvi),ckamuzuvi@umat.edu.gh (C. K. Amuzuvi)

\section{To cite this article:}

Christian Kwaku Amuzuvi, Fuseini Mumuni. Investigating the Effect of Increased Heatsink Temperature and Packaging-Induced Strain in a Single Emitter Laser Using a Laser Diode Simulation/Emulation Tool. Journal of Electrical and Electronic Engineering. Vol. 1, No. 4, 2013, pp. 73-78. doi: 10.11648/j.jeee.20130104.12

\begin{abstract}
This paper explores further the capability and versatility of Barlase in establishing deeper understanding of the behaviour of an emitter in a laser bar. The interactions between an emitter and the substrate upon which it is mounted are important in learning more about the degradation process that occurs in lasers. It is well known that various factors come into play in the operation of individual emitters and full laser bars (L-I characteristics, threshold, efficiency, etc.) but one of the most important is the effect of increased heatsink temperature and packaging-induced strain in laser bars. Barlase is therefore used to investigate these effects based on the Arrhenius equation. The relevant parameters are updated at each aging step. Barlase allows a better understanding of how current competition, temperature and the level of defects affect the output power and the degradation rate of a bar.
\end{abstract}

Keywords: By-Emitter, Emitter, Quantum Well, Defect, Heatsink Temperature, Non-Radiative Recombination, Packaging-Induced Strain, Degradation, Temperature, Threshold Current, Slope Efficiency

\section{Introduction}

High power semiconductor laser diodes have occupied the minds of researchers in the last decade due to the emerging widespread usage in the fields of medicine, industry and in telecommunications [1-3].

The effectiveness of Barlase has already been demonstrated using hypothetical laser bars and published elsewhere [4]. In this paper however, Barlase is being used to simulate/emulate degradation processes using a hypothetical single emitter high power laser bar considering the effect of increased heatsink temperature and that of packaging-induced strain independently in laser bars to ascertain their individual effects.

This paper gives a further credence to the by-emitter degradation analysis technique developed over recent years Xia et al. [5], [6], Bull et al. [7], [8] and Bream et al. [9]. This tool is also an addition to the by-emitter analysis technique where the effects of certain factors that affect the degradation of laser emitters/bars can be investigated. The objective of this study is to investigate the effect of increased heatsink temperature and packaging-induced strain independently in laser bars to ascertain their individual effect on the degradation of a semiconductor laser bar. The model has been validated using experimental results in a publication elsewhere [10].

\section{Materials and Methods}

The standard test structure selected is one employed in the experimental work in a task published elsewhere [10]. This structure was selected bearing in mind the fact that an attempt will be made to further emulate the degradation of bars made from "the same epitaxial" structure with similar dimensions. The structure used was the $975 \mathrm{~nm}$ narrow-angle $\left(<1^{\circ}\right)$ tapered laser from Alcatel Thales III-V Lab. The total length was $2.4 \mathrm{~mm}$, consisting of a $200 \mu \mathrm{m}$ ridge waveguide and a $2200 \mu \mathrm{m}$ tapered amplifier. The front and rear facet reflectivities were $3 \%$ and $90 \%$, respectively. The 'standard' simulation of this structure assumes a heatsink temperature of $300 \mathrm{~K}$ and a trap density in the 
quantum well (QW) of $2 \times 10^{15} \mathrm{~cm}^{-3}$ [11]. All of the simulations in this paper use this structure and the results are referenced to this 'standard' structure. Figure 1 shows the laser structure.

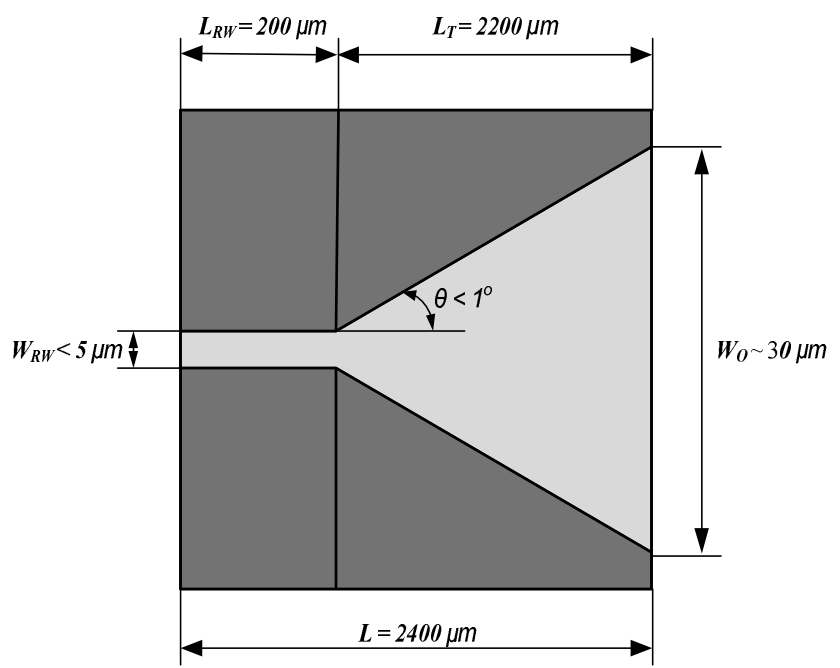

Figure 1. A hypothetical standard $975 \mathrm{~nm}$ tapered laser structure.
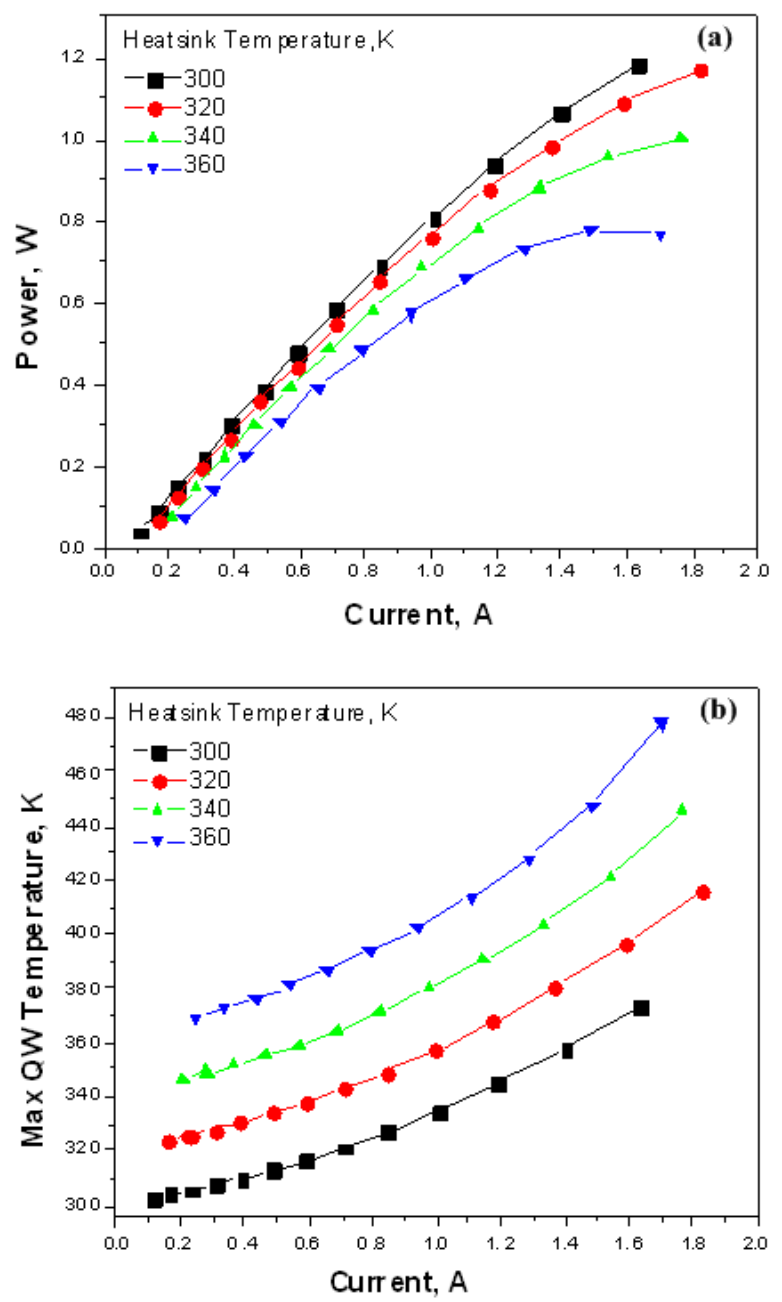

Figure 2. (a) Power-current characteristics (b) the maximum temperature in the $Q W$ as a function of current.
Simulations were performed considering the effect of increased heatsink temperature and considering the effect of packaging-induced strain in laser bars [12]. The data obtained from these single emitter simulations were performed in the constant current mode of operation [10].

\section{Results and Discussion}

\subsection{Effect of Increased Heatsink Temperature}

To investigate the effect of elevated heatsink temperatures (relative to $300 \mathrm{~K}$ ), simulations were performed at heatsink temperatures of $320 \mathrm{~K}, 340 \mathrm{~K}$ and $360 \mathrm{~K}$. Figure 6 shows the power-current characteristics and the evolution of the maximum QW temperature with bias current for each heatsink temperature. As expected, the P-I curves show an increase in threshold current, a decrease in power and a drop in slope efficiency as the heatsink temperature is increased. Thermal roll-over is observed at high injection currents for all heatsink temperatures. As the heatsink temperature is increased, the point at which thermal roll-over begins moves to lower currents.
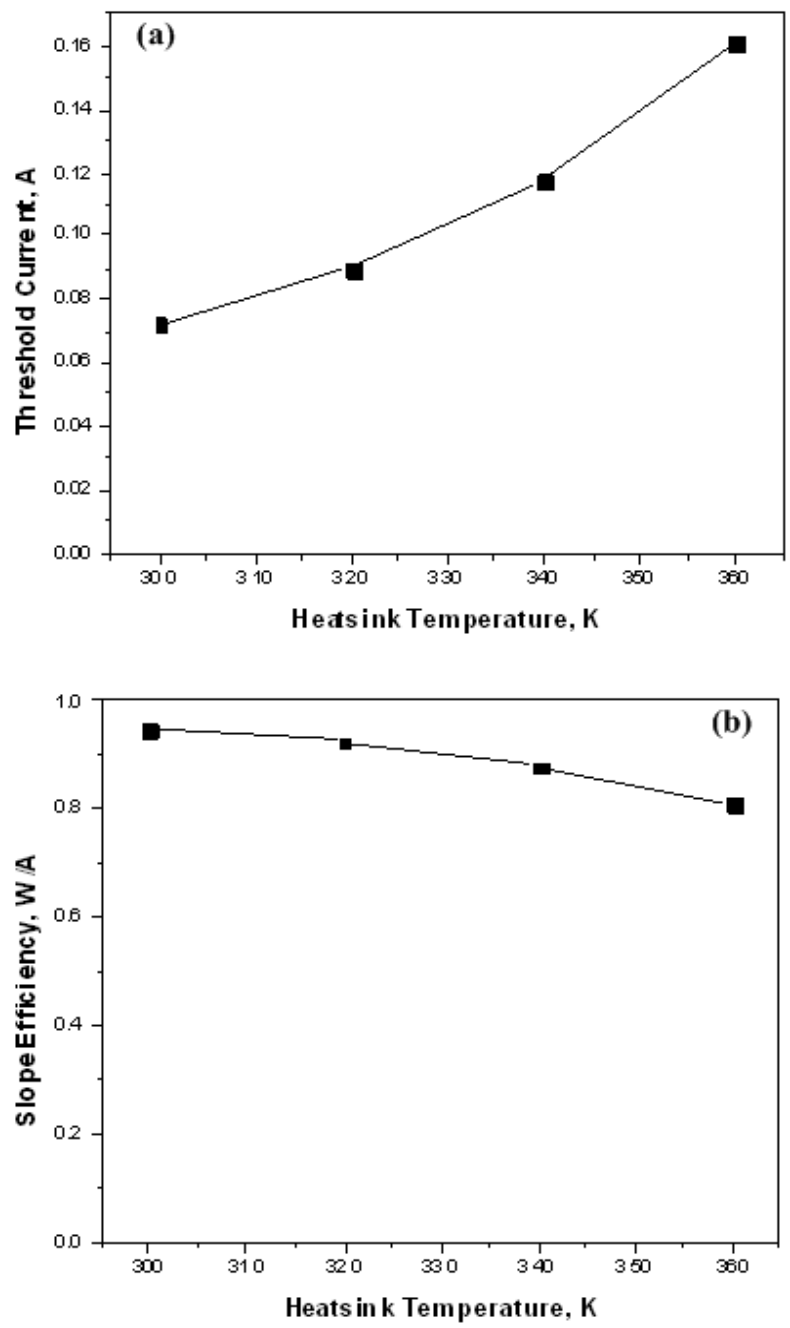

Figure 3. (a) Graphs showing the dependence of threshold current and (b) efficiency on heatsink temperature. 
From the P-I curves in Figure 2, the threshold current and slope efficiency have been extracted and these are plotted as a function of heatsink temperature in Figure 3. As expected, an increase in the threshold current and a decrease in the slope efficiency are observed as the temperature of the heatsink increases.
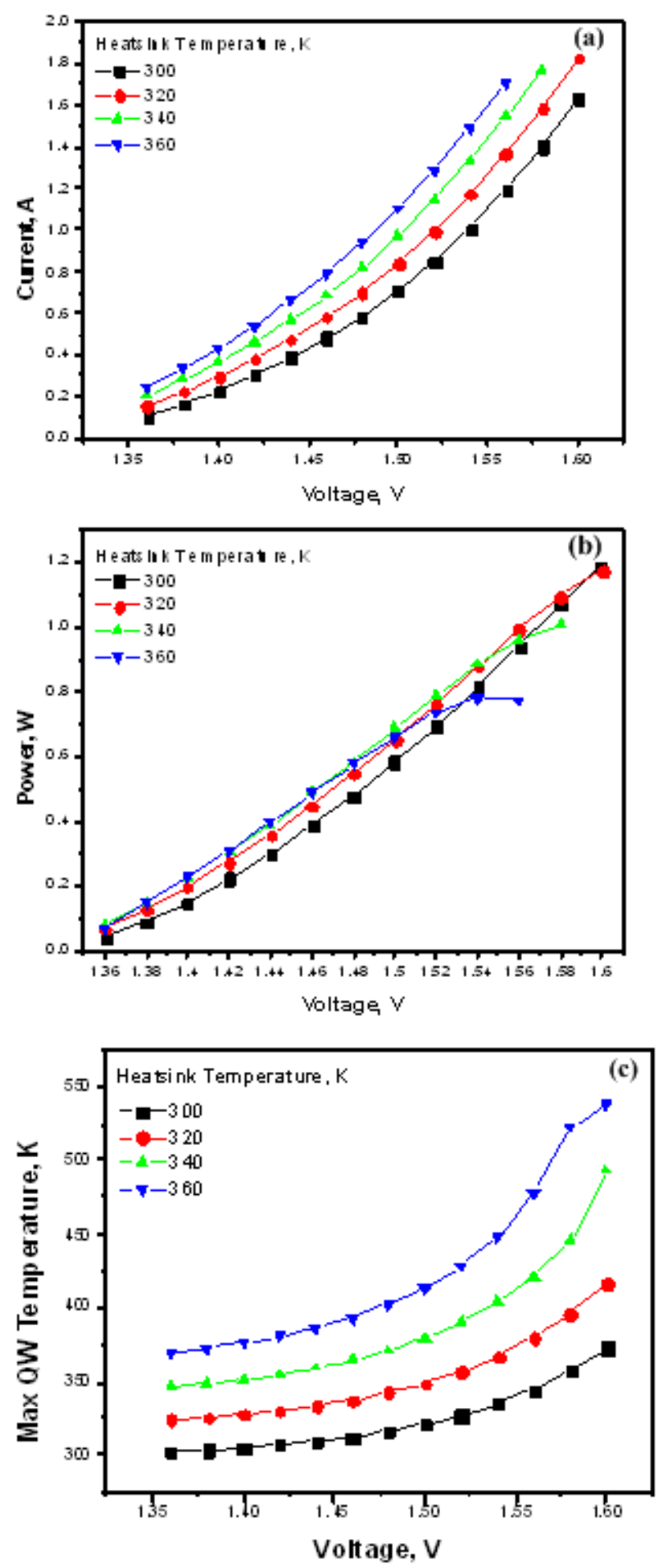

Figure 4. (a) Current-voltage characteristics, (b) power-voltage characteristics, and (c) the maximum temperature in the $Q W$ versus voltage for simulations at different heatsink temperatures.
Increasing the temperature of an emitter from $300 \mathrm{~K}$ to $320 \mathrm{~K}$, results in an increase of nearly $25 \%$ in the threshold current. For an emitter at $360 \mathrm{~K}$, the threshold current has more than doubled. Clearly, even a small increase in temperature can have a significant impact on the threshold current. The corresponding decreases in slope efficiency are $\sim 2.5 \%$ for an emitter at $320 \mathrm{~K}$ and $15 \%$ for an emitter at 360 K.

This analysis can be misleading, however, since the emitters in a bar are biased with a constant voltage, not a constant current. Figure $4 \mathrm{a}$ shows the current-voltage and power-voltage Figure $4 \mathrm{~b}$ characteristics for these simulations at different heatsink temperatures. Over the range of heatsink temperatures investigated here, the current can increase by as much as $50 \%$ for a fixed voltage of $1.50 \mathrm{~V}$. From the P-V curves, the voltage at which thermal roll-over begins is clearly identifiable. For example, in a laser bar at $1.50 \mathrm{~V}$ (Figure $4 \mathrm{~b}$ ), emitters at $300 \mathrm{~K}$ and $320 \mathrm{~K}$ would be operating in their normal operating regimes, but emitters at $340 \mathrm{~K}$ would be at the onset of thermal roll-over and emitters at $360 \mathrm{~K}$ would be operating well past the onset of thermal roll-over.
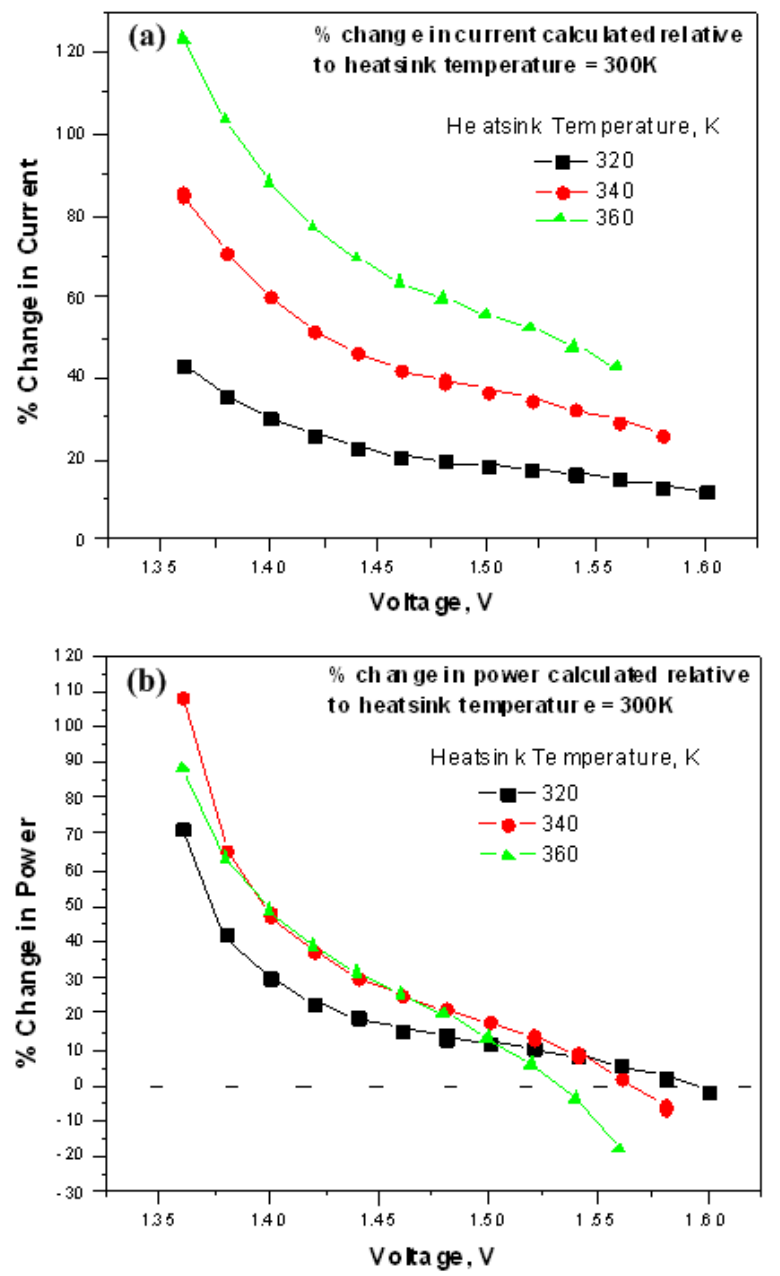

Figure 5. (a) Graphs showing the percentage change in current and (b) power in the simulations at different heatsink temperature relative to the standard heatsink temperature of $300 \mathrm{~K}$. 
The percentage change in both bias current and output power (relative to a heatsink temperature of $300 \mathrm{~K}$ ) are shown in Figure 5 as a function of bias voltage. These graphs show the dramatic percentage increases in current and power as the temperature rises for emitters biased with a fixed voltage. These figures show that current competition becomes an important effect in laser bars where there is an uneven distribution of temperatures between the emitters (a very typical situation).
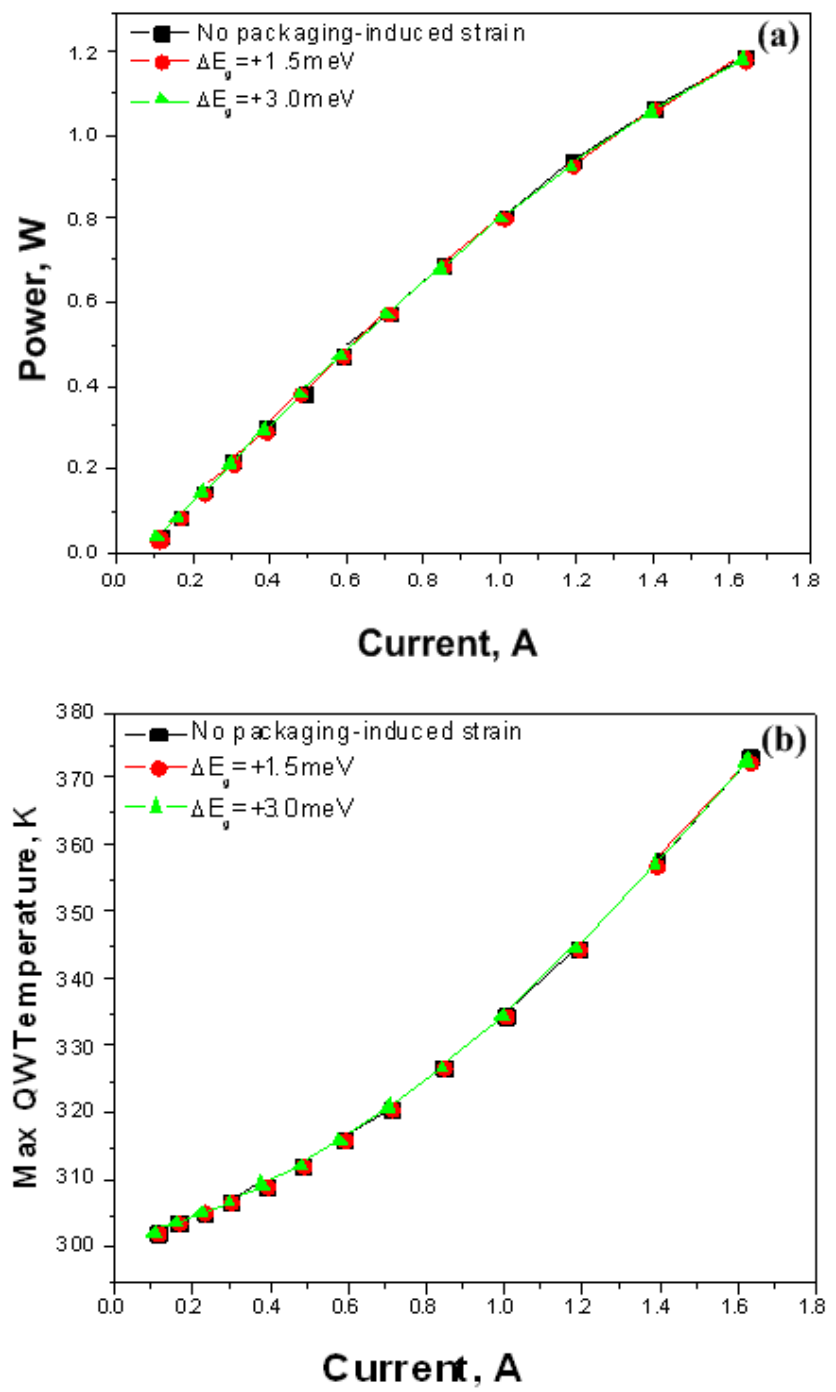

Figure 6. (a) Power-current characteristics and (b) the maximum temperature in the $Q W$ as a function of current for simulations with different levels of packaging-induced strain.

\subsection{Effect of Packaging-induced Strain}

To investigate the effect of packaging-induced strain, simulations were performed where the QW bandgap energy was increased by $1.5 \mathrm{meV}$ and $3.0 \mathrm{meV}$ to emulate medium and high levels of packaging-induced strain. In order to do this, the gain spectra used in Speclase [4] (including differential gain) were recalculated for each of the bandgap energies (strains) investigated. The values investigated were chosen based upon experimental measurements made on a wide range of packaged laser bars. The typical experimentally measured increases in the QW bandgap energy are in the range of $1 \mathrm{meV}$ to $5 \mathrm{meV}$ (Xia et al. [5], [13] and Bull et al. [7]), where a $5 \mathrm{meV}$ shift corresponds to a compressive strain of $\sim 0.12 \%$ [6]. Figure 6 shows the power-current characteristic and the evolution of the maximum QW temperature with bias current for each of the packaging-induced strain levels investigated. A reduction in the bandgap energy is expected to affect the turn-on voltage of the laser diode. Thus, even though the power-current curves show very little change as a function of strain, the higher strain conditions do indeed cause changes to the operation of a laser biased at a fixed voltage, as shown in Figure 7.

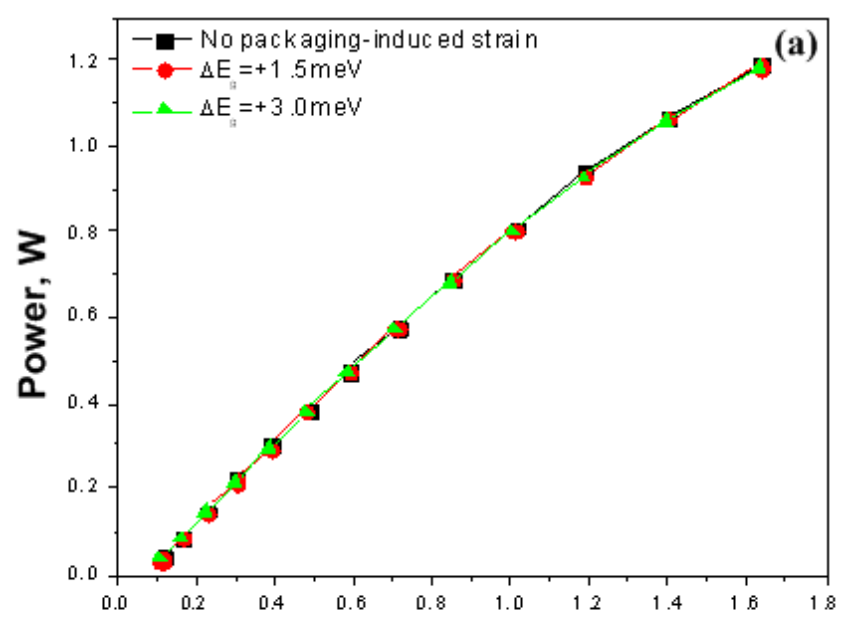

Current, A

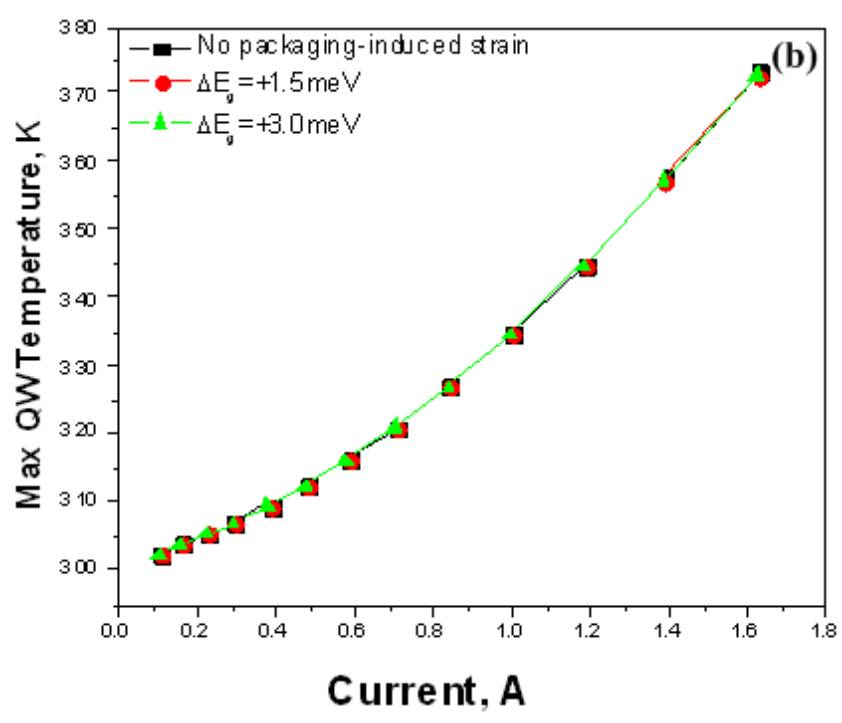

Figure 7. (a) Power-voltage characteristics and (b) the maximum temperature in the $Q W$ as a function of voltage for simulations with different levels of packaging-induced strain.

As with the previous effect, to see the differences in the simulations, the percentage change (relative to the case where there is no packaging-induced strain) in both bias current and output power are shown as a function of bias 
voltage in Figure 8. From these figures, it is possible to see the differences in the laser operation that are caused by increasing the level of packaging-induced strain. For a bias voltage of 1.45-1.50 V, a high level of packaging-induced strain can reduce the current by up to $1 \%$ and the power by up to $2 \%$.
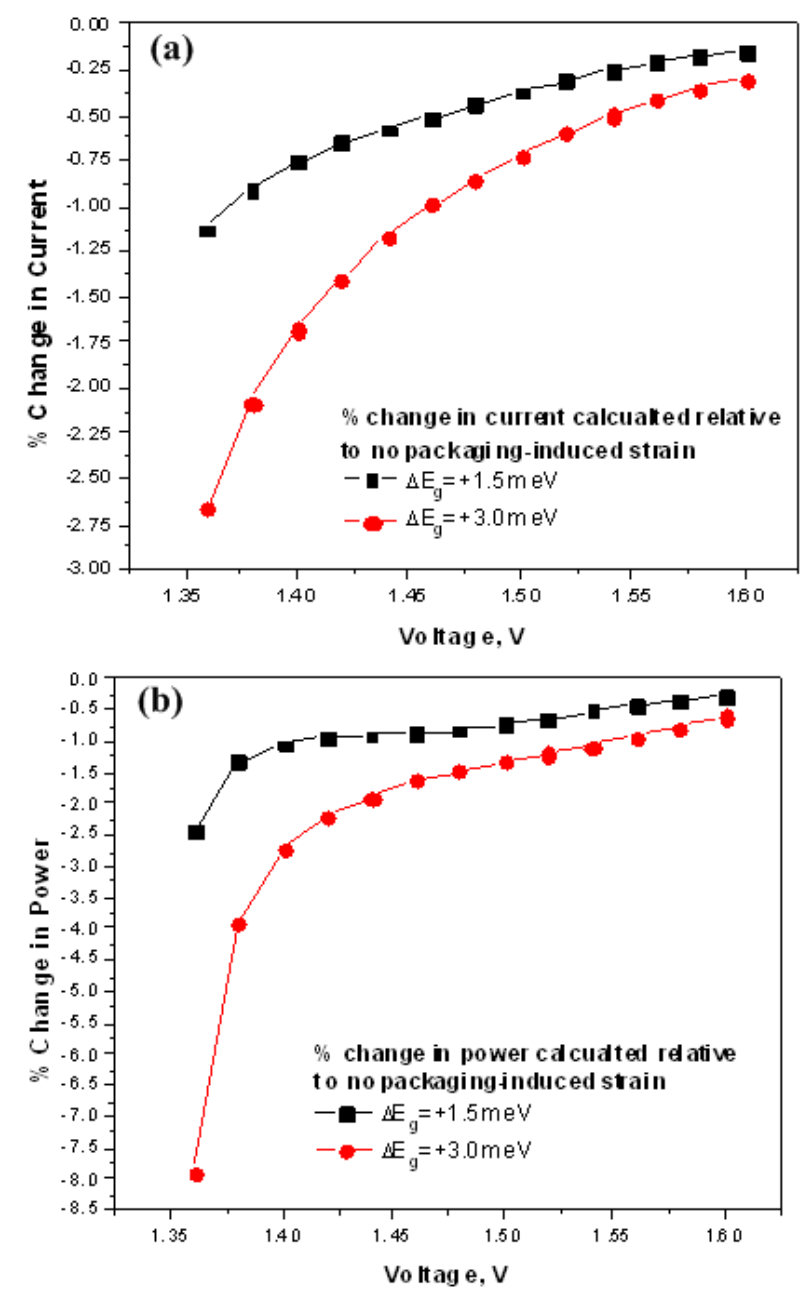

Figure 8. (a) Percentage change in current and (b) power in simulations at different levels of packaging-induced strain relative to the standard case of no packaging-induced strain.

The principal factors that affect laser degradation are defects (both point defects and line defects), temperature, packaging-induced strain, and inhomogeneous heatsinking (e.g. solder voids, solder flow, etc.). To understand how each of these factors affects the operation and degradation of a laser, a number of simulations were performed using a single emitter bar. In this paper, investigations were done considering the effect of increased heatsink temperature and that of packaging-induced strain independently.

The simulations were performed for a constant bias voltage, as this is the common factor between individual emitters once they are part of a laser bar. In all cases, simulations were attempted at voltages ranging from $1.32 \mathrm{~V}$ to $1.60 \mathrm{~V}$ at intervals of $0.02 \mathrm{~V}$. Majority of these simulations converged within $10-15$ round trips.

\section{Conclusion}

The results presented in this paper show that even simulations of single emitters can provide a great deal of insight into how these emitters will operate in the context of a laser bar. Nonetheless, it is important to consider their operation with respect to a fixed bias voltage and not with respect to a fixed bias current. The two factors investigated here (temperature and strain) all affect the operation of an emitter. Unsurprisingly, temperature is found to provide the most important degradation effect. Indeed, it is well known that the nucleation, propagation and growth of defects can be increased by temperature. Thus, the rates of defect generation and propagation within a bar are inextricably linked to the temperature profile. The effects on laser operation of packaging-induced strain are small, but this is nonetheless important when combined with all the other factors that can affect laser degradation. This research is ongoing and more will therefore need to be done to especially determine the effect of temperature on the degradation of lasers into more detail.

\section{Acknowledgment}

CKA thanks the University of Mines and Technology, Tarkwa, Ghana and the GetFund for their support.

\section{References}

[1] Schulz, W. and R. Poprawe, 2000. "Manufacturing with novel high-power diode lasers", IEEE Journal of Selected Topics in Quantum Electronics, vol. 6, pp. 696-705.

[2] Gannot, I and R. W. Waynant, 2001. "Introduction to the issue on lasers in medicine and biology", IEEE Journal of Selected Topics in Quantum Electronics, vol. 7, pp. 873-873.

[3] Rochat, E., K. Haroud, and R. Dandliker, 1999. "High-power Nd-doped fiber amplifier for coherent intersatellite links", IEEE Journal of Quantum Electronics, vol. 35, pp. 1419-1423.

[4] Amuzuvi, C. K. and J. C. Attachie, 2013. "Describing a Laser Diode Emulation Tool Using Single Emitter Simulation Results". Research Journal of Applied Sciences, Engineering and Technology, 5(04): 1358-1361.

[5] Xia, R., E. C. Larkins, I. Harrison, S. R. A. Dods, A. V. Andrianov, J. Morgan and J. P. Landesman, 2002. "Mounting-induced strain threshold for the degradation of high-power AlGaAs laser bars", IEEE Photon. Technol. Lett., 14, 893 .

[6] Tomm J. W., A. Gerhardt, T. Elsaesser, D. Lorenzen, and P. Hennig, 2002. "Simultaneous quantification of strain and defects in high-power diode laser devices", Applied Physics Letters, vol. 81, pp. 3269-3271.

[7] Bull S., J. W. Tomm, M. Oudart, J. Nagle, C. Scholz, K. Boucke, I. Harrison, and E. C. Larkins, 2005. "By-emitter degradation analysis of high-power laser bars", Journal of Applied Physics, vol. 98, p. 063101. 
[8] Lim, J. J., T. M. Benson and E. C. Larkins, 2005 "Design of wide-emitter single-mode laser diodes", IEEE J. Quantum Electron., 41, 506.

[9] Bream, P. J., J. J. Lim, S. Bull, A. V. Andrianov, S. Sujecki and E. C. Larkins, 2006. "The impact of nonequilibrium gain in a spectral laser diode model", Opt. Quantum Electron., 38, 1019 .

[10] Amuzuvi, C. K., 2013. Emulation and By-Emitter Degradation Analysis of High Power Lasers: Lap Lambert Academic Publishing, Saarbrücken, Germany, ISBN 978-3-659-37035-9.
[11] Agrawal G. P. and N. K. Dutta, 1993. Semiconductor Lasers: Kluwer Academic Publishers; 2nd Revised edition edition.

[12] Amuzuvi, C. K., Bull S., Tomm J. W., Nagle J., Larkins E. C., Sumpf B., Erbert G., Michel N., and Krakowski M., 2011. "The impact of temperature and packaging-induced strain on current competition and emitter power in laser bars" Applied Physics Letter.

[13] Bull S., 2004. "Photo- and Electroluminescence Microscopy and Spectroscopy Investigation of High Power and High Brightness Semiconductor Laser Diode", Dissertation, University of Nottingham, United Kingdom. 